\title{
Predictive Diagnosis of Breast Cancer Using Deep Neural Network
}

\author{
T. Subha ${ }^{1}$, N. Susila ${ }^{2}$, R. Ranjana ${ }^{3}$, R. Anushya ${ }^{5}$ and S. Amritha ${ }^{6}$ \\ ${ }^{1}$ Associate Professor, Department of Information Technology, Sri Sairam Engineering College \\ ${ }^{2}$ Professor and Head, Department of Information Technology, Sri Krishna College \\ of Engineering and Technology \\ ${ }^{3}$ Associate Professor, Department of Information Technology, Sri Sairam Engineering College \\ 4,5Students, Department of Information Technology, Sri Sairam Engineering College
}

\section{ABSTRACT}

The ultimate aim of the study was to predict the overall survival rate for women affected by breast cancer by developing and validating a prognostic model. In this study mammography image were collector and the health records for at least 1 year before mammography. The algorithm was trained on mammogram and health records of women to make two breast cancer predictions: (i)to predict biopsy malignancy and (ii)to differentiate normal from abnormal examination. In this model,ANN's were used to complete the task. This model was developed using a large proportion of concealed layers for thedatageneralization.Alargeproportionclosetoa1000mammographyrecordsandresultswere used in the model to differentiate between benign and malignant tumors. Before being inputted, all the data was inspected by radiologists. The accuracy of the model was determined to be an AUC of 0.96 and was a huge success(area or curve used to determine the success of model). This model is highly consistent, effective and less prone to error when compared to diagnosis performed by pathologists. The study obtained very high accuracy of predictions such as 93\% and 95\%. This facilitates early and accurate diagnosis of breast cancer for patients and early diagnosis is integral in cancer diagnosis. This also contributes as a useful tool for new selective drugs for these tumors to be treated. In Today World, Technology has facilitated great improvements in various important aspects of the world such as healthcare, agriculture, business etc. Despite this tremendous growth and improvement various diseases are still on the rise world wide. Among these Cancer stands as one of the major causes of death and accounts for about 9.6million deaths world wide. Cancer has various forms. Among which Breast Cancer is one of the most dangerous and common reproductive cancers that mostly affects women. According to the World Health Organization, Breast cancer affects about 2.1 million women every year. In order to improve Breast cancer outcome and survival, Early detection is critical. Hence, the goal of our project is to use Machine learning techniques and deep learning to increase the proportion of breast cancers identified at an early stage, allowing for more effective treatment to be used and reduce the mortality rate. Since early detection of can ceriskey to effective treatment of breast cancer, we use various machine learning and deep learning techniques to predict if a tumour is benign or malignant, based on the features provided by the data in a more accurate,consistent and less prone toerror.

KEY WORDS: BREAST CANCER, WORLD HEALTH ORGANIZATION, 2.1 MILLION WOMEN EVERY YEAR.

Biosc Biotech Res Comm P-ISSN: 0974-6455 E-ISSN: 2321-4007

\section{crossef}

Identifiers and Pagination

Year: 2021 Vol: 14 No (5) Special Issue

Pages: 218-224

This is an open access article under Creative

Commons License Attribn 4.0 Intl (CC-BY).

DOI: $h$ ttp://dx.doi.org/10.21786/bbrc/14.5/40

\section{INTRODUCTION}

The occurrence of mutations which are changes in genes regulating the growth of cell causes the deadly disease Cancer. This causes the multiplication and division of cells in an uncontrollable fashion. The cancer developed in the breast region is defined as breast cancer. The formation of cancer takes place in the duct or lobules of breast.

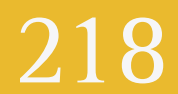


The milk-producing glands are termed as lobules and the milk transportation pathways to the nipple from the glands are known as ducts. Occurrence of cancer can be extended to the connective fibrous and fatty tissue of the breast. The affected cancer tissue has the dangerous ability to affect other healthy tissues and also spready or travel to the lymph nodes in the arm. The movement of the cancer cells to the rest of the body parts is facilitated by the lymph nodes.

Machine learning (ML) and its techniques have recently provided great results in the healthcare field. Although Machine learning models are not new and have been increasingly used analyse data in health care, A machine learning can be used to diagnose breast cancer at a level equal to pathologists and Hence acceptable in clinical practice as a very useful tool. The purpose of our study is to use Machine learning and deep learning for early breast cancer prognosis and assess its stage and survival rate of the patient. Analyse data in health care, A machine learning can be used to diagnose breast cancer at a level equal to pathologists and Hence acceptable in clinical practice as a very useful tool. The purpose of our study is to use Machine learning.

Breast Cancer: The cancer occurred and developed in the region of the Breast is termed as breast cancer. Typically, Breast cancer occurs in women and also in men in rare scenarios. It is one of the most prevalent and deadly diseases around the world. It is said to commonly occur in women between the age group 40-55 years old. Breast cancer is said to initiate with cells in the best growing in an uncontrollable manner. Early diagnosis and treatment is key in breast cancer.

Symptoms: One of the dangerous attributes of breast cancer is that close to no symptoms are visible during its early stages. There are many scenarios in which the tumor being very small can be neglected, However the abnormality can be identified on a scan or mammogram. When the tumor is first felt it mostly mean sit'sanewone formed in the breast which was initially absent. But, all tumors in the breast are not a tumor caused by cancer. A variety of symptoms are caused due to each kind of breast cancer. Most of these symptoms are similar in character, but there are few symptoms which are distinct. The common symptoms of breast cancers include:

- A lump in the breast developed recently and may or may not cause pain.

- Pain in breast region

- Skin turning red in the breast

- $\quad$ swelling in breast region

- discharge from nipple except breast milk

- discharge containing blood from nipple

- Skin flaking or peeling nipple or breast region.

- Change in size or shape of breast which takes place suddenly.

- Nipple inversion

- Appearance changes on breast region

- Under arms welling
Types of Breast Cancer: The common types of breast cancer are as follows:

In situ Cancers: This type of cancer does not spread to other regions and stays in the region of initial development.

Ductal carcinoma in situ (DCIS): The carcinoma at the very initial stage.(stage0).The cancer hasn't spread and in its initial region of development. However, if untreated this may become dangerous. This stage of cancer is easily curable. Lobular carcinoma in situ (LCIS). This carcinoma originates exclusively in lobules producing milk. It isn't termed cancer but has the ability to develop into breast cancer in the future. Regular medical checkups are mandatory.

Invasive Cancers: These are dangerous and have the ability to grow and spread across the body.

Invasive or infiltrating ductal carcinoma (IDC): This originates in the ducts producing milk and grow and damage the surround cells and tissues in the breast region by breaking the walls. It is highly prevalent and accounts for a greater margin of deadly cases.

Invasive lobular carcinoma (ILC): This originates in the lobules and spreads to the other parts It accounts for about lesser margin of deadly breast cancers.

Breast Cancer Risk Factors: The reason and cause of breast cancer is yet to be identified. But, The probability of occurrence can be determined by some factors such as age, genetic factors, personal health history, and food habits contribute to them. The factors of risk are classified as controllable and Uncontrollable risk factors.

\section{Breast Cancer Uncontrollable Risks factors:}

- Age: Women below the age are less likely to be in risk and womenaboveage50areproneto the disease.

- Dense breasts: Presence of more connective tissue than fatty tissue can reduce the visibility of tumors in the breast in scans.

- Personal history of cancer: This plays a major role as previous breast cancer patients are more prone to recurrence of the disease.

- Family history: If a close blood relative had breast cancer, you're more prone to the disease.

- Genes: Mutations or change in genes may cause this disease. Especially genes such as, BRCA1 andBRCA2.

- Menstrual history: A patient is more prone to breast can cerif:

1. Early periods initiation before the age of 12 .

2. Periods don't stop until age55.

\section{Breast Cancer Stages}

- Early stage (stage 0) or non-invasive breast cancer. The presence of the disease is completely limited to its place of origin and cause no symptoms.

- Stage I breast cancer. The tumor or cancer is very 
small in size and doesn't spread but stay in its original position.

- $\quad$ Stage IIA breast cancer. The tumor is:

- less than $2 \mathrm{~cm}$ with development in underarm.

- Bigger than $2 \mathrm{~cm}$ and smaller than $5 \mathrm{~cm}$ with no development any where else.

- Stage IIB breast cancer. A tumor that's:

- A big tumor of $5 \mathrm{~cm}$ in size without any development in any other region.

- A big tumor in size with development in the other regions across breast.

- $\quad$ Stage IIIA breast cancer or locally

\section{advanced breast cancer:}

- A large tumor in size and spread across the region of breast such as lymph nodes under the arm.

- A cancerous tumor that spreads to surrounding tissues.

- $\quad$ Stage IIIB breast cancer. A tumor which spreads across the chest.

- $\quad$ Stage IIIC breast cancer. A tumor which has rapidly spread and includes development in lymph nodes

- Stage IV (metastatic) breast cancer. A tumor which rapidly spreads from its place of origin to other parts of the body and is very dangerous.

\section{MATERIAL AND METHODS}

Dataset: The dataset consists of 292,624 mammography examinations screened digitally from close to 2 lakh patients. The examination distinctly includes minimum 4 images which were a part of the mammography screening. The four images are as follows: L-CC (left craniocaudal), R-CC (right craniocaudal), L-MLO (left mediolateral oblique) and R-MLO (right mediolateral oblique). The reports obtained from the pathology department were used in the process of determining the labels which designate whether the tumor in the breast denotes malignant tumor or benign tumor.

In a period of 140 days of mammogram screening close to 5000 examinations accompanied by a minimum of one biopsy was performed. It was found that 980 $(8.2 \%)$ were found to be malignant cancers and close to $5000(47 \%)$ were determined to have benign cancer and the remaining $200(20 \%)$ were determined to have both benign and malignant cancer. The proportion remaining that failed to match with the results of the biopsy, were labeled as malignant and benign tumours absent in both breasts. The examinations which were found to be matching with the results of biopsies, were provided to pathologists and radiologists to determine at pixellevel placement or place of origin of the biopsied lesions. It was then determined by the specialists that about $32 \%$ of the examinations were concealed and failed to be visible or found on mammography and could be determined of their presence using other imaging techniques such as: MRIor Ultrasound.

\section{METHODOLOGY}

Adaptive mean filter has been used to remove noise from image. Since it is better among all the spatial filters and distinguish fine details from noise. The Adaptive Median Filter is used to determine the noise impulse affected pixels in an image by spatial processing. The pixels are classified by adaptive mean filters as noise by comparing each pixel in the image to its surrounding neighbor pixels. The size of the neighborhood is adjustable, as well as the threshold for the comparison. A structurally disjoined pixel to its similar pixels and greatly varying from a maximum of the pixels surround it is designated as impulse noise.

The median pixel value is then exchanged by the noise pixels in the surroundings where pixels have already passed the noise labelling test. Initial conversion of the image into grayscale image using rgb2gray () function which is followed by the application of adaptive mean filtering to the resulting image and then finally converting the image into unsigned integer 8 using uint8() function. In this manner the image is preprocessed and followed by performing GMM segmentation (Gaussian Mixture Model)onthepreprocessedimagewith2regionsand 2 components GMM components 2 and maximum number iterations 10 . we performed k-means segmentation.

with $\mathrm{k}=2$. then we Implemented HMRF-EM (Hidden Markov Random Field Model) and its ExpectationMaximization Algorithm.

\section{Block Diagram}

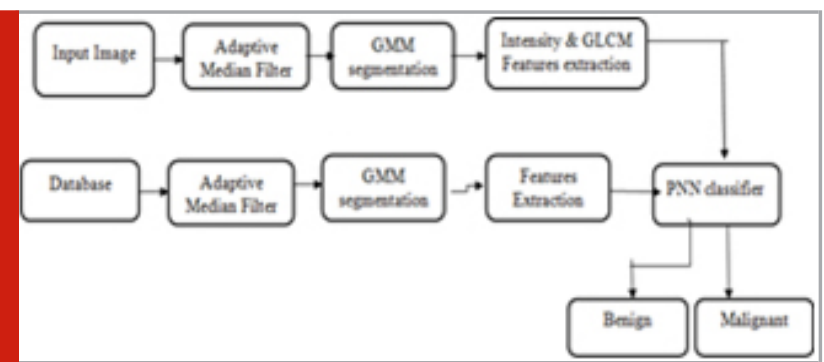

Figure 2.A: Diagrammatic representation of breast cancer examination classification as a learning task.

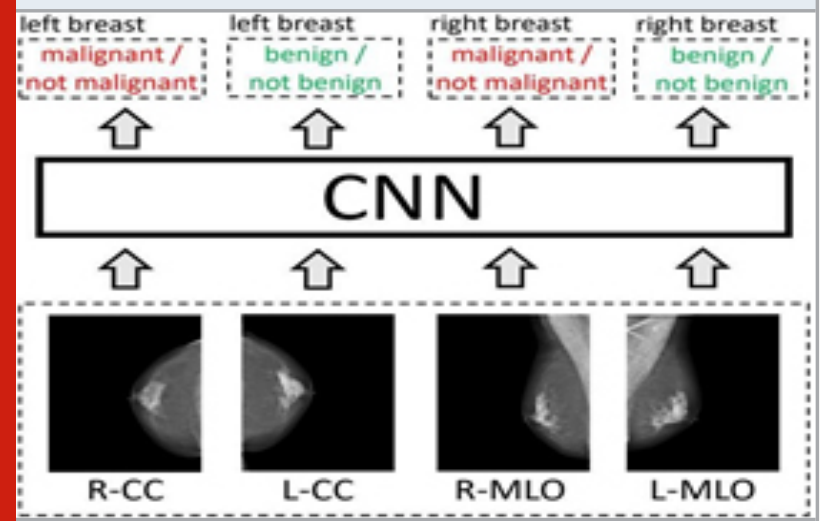

Deep CNNs for cancer classification: Two primary binary labels were designated for every breast under examination: malignant tumor present/absent in the breast region, and the benign tumor presence/absence 
in the breast region.Taking into account both the sides of the breast,left and right every examination consists of four labels. Our ultimate goal is to construct four distinct predictions which are attributed to the four distinct labels corresponding to every examination. The four High resolution images are in agreement with the views of the four mammography screening are taken as the input. Every image is cropped to a fixed size of 2767 $\times 1924$ pixels for CC views and $2947 \times 1784$ pixels for MLO views.

Model Architecture: We trained a deep multi-viewCNN of architecture. There are 2 core modules in the overall network:

1. Four columns four view-specific, each based onthe ResNet architecture that outputs a fixeddimension hidden representation for each mammographyview,

2. Mapping of the calculated concealed representations to the final predictions using two completed and connected layers. The calculation of 256-dimension concealed representation vector of every view was performed using Resnet-22. The weights were shared by columns applied to L-CC/R- CC.Thisw asalsofollowedandweightsweresharedby columns applied to L-MLO/R-MLO. This step is followed by the concatenation process of L-CC and R- CC representation into a 512 dimensional vector, and the generation of predictions of the four outputs are achieved by application of two completely connected layers.This process is repeated for the successive L- MLO and R-MLO views. The final output and predictions are deduced by using the average probability of CC and MLO branches of the model.

Figure 3: Architecture of our model. Four Res Net-22 columns take the four views as input

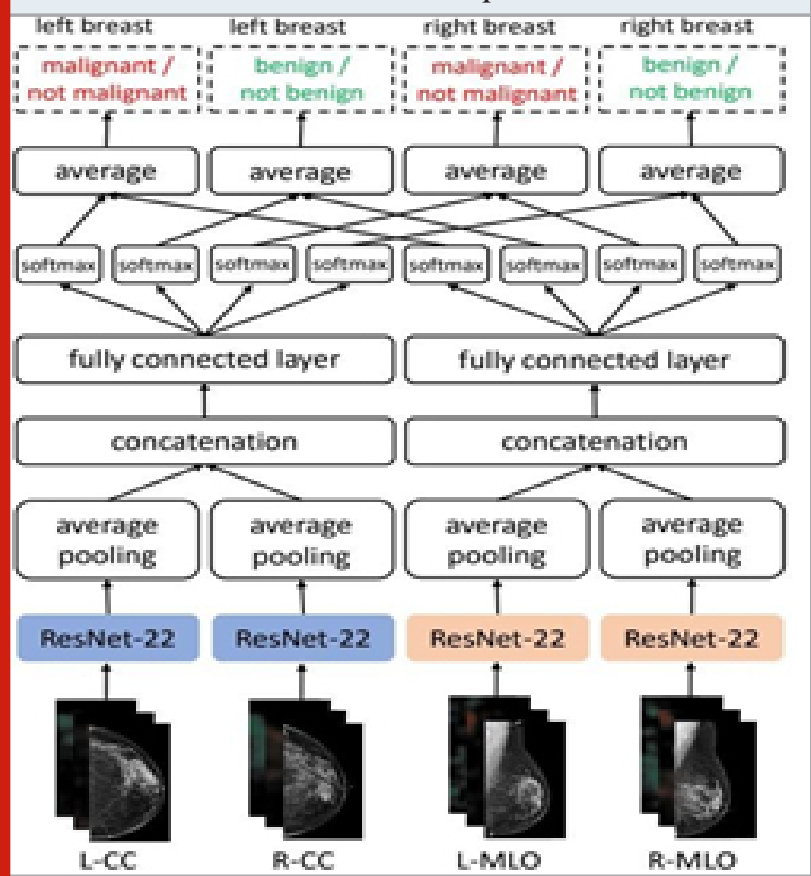

Fig.3. Architecture of our model. Four Res Net-22 columns take the four views as input. The architecture is classified into two branches called CC and MLO. In each branch, the corresponding right and left representations from the ResNets are individually average- pooled spatially and concatenated, and two fully connected layers areapplied to compute the predictions for the four outputs. The predictions are averaged between the CC (craniocaudal) and MLO (mediolateral oblique) branches. Weights are shared between L- CC/RCC columns and L-MLO/R-MLO columns. The first layer of the columns are modified in accordance to inputs as additional channels are added to theheatmaps.

Experiments: For each and every experiment, the training set is used for optimization of parameters of our model and the validation set and thetraining procedure. Unless in any other cases specified, the results are computed throughout the screening population. To acquire, test sample prediction for each sample we applied random transformationsforinput10times,appliedthemodelto 10 of the samples each separately and then average the 10 predictions (in SI details). To improve our results further, we used the technique of model ensemble [19], wherein the predictions of various different models are averaged to produce overall prediction of the ensemble. In our case, we trained 5 copies of every model with different random initializations of the weights within the fully connected layers. The pre-trained model's weights are used to initialize the remaining on BIRADS classification, it gives our model a significant boost in performance. For each model, we report the results from a single network and from anensemble.

\section{Test Populations}

In the experiments, to test the various hypothesis we evaluate our model on several populations:

1. screening population, it includes all exams from the test set without sub sampling;

2. biopsied subpopulation, which is subset of the screening population, and includes the exams from the screening population containing breasts which underwent abiopsy;

3. reader study subpopulation, consists of the subpopulation of the biopsied report and randomly sampled subset exams from the screening population without any findings.

Evaluation Metrics: The models were initially evaluate in terms of AUC (area under the ROC curve) for malignant or not malignant and benign or not benign classification tasks on the breast level. The model and readers' responses on the subset for reader study are evaluated in terms of AUC and precision-recall AUC (PRAUC), which are the regularly used evaluation metrics of the performance of the radiologists.

The different as pect of predictive model performanceis captured by ROC and PRAUC. The RO Ccurve summarizes the trade-off between the true positive and false positive rate for a model using probability thresholds. The true positive rate and positive predictive value is summarized 
by the precision recall curve for a model using different probability thresholds.

Figure 4: AUCs for patients grouped by age and by breast density

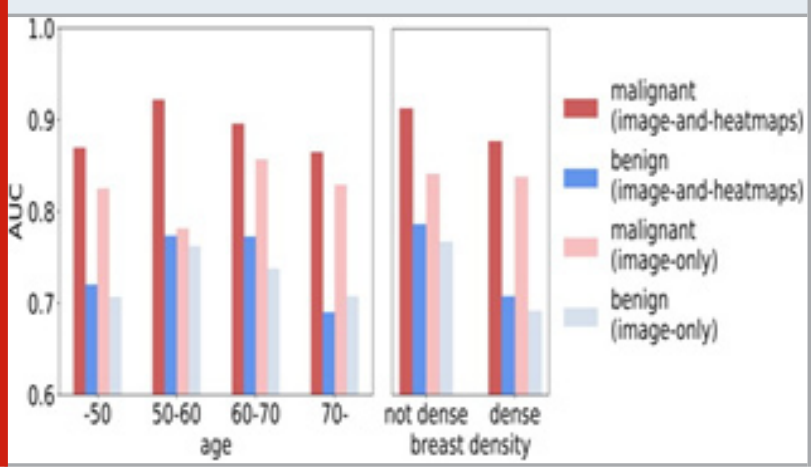

Results across ages and breast densities: We split the test data by patient age, breast density and evaluate the model for each and every subpopulation (Fig 4). The performance of the image-only and image- and heat maps model varies based on age. We also find that both models perform worse on dense breasts ie..in "het erogeneouslydense"and "extremely dense"thanon fattier ones which are almost entirely fatty, which is consistent with the decreased sensitivity of radiologists for patients with dense breasts. Differences between the model's performance whether its benign/notbenign classification is larger than in malignant/not malignant classification. We conclude that this is due to age and the level of noise is influenced by the breast density in benign/not benign labels, associated with radiologists confidence in their diagnoses.

\section{Literature Survey}

\begin{tabular}{|c|c|c|c|c|}
\hline S.N 0 & $\begin{array}{l}\text { NAME OF } \\
\text { THE PAPER }\end{array}$ & AUTHOR & $\begin{array}{l}\text { DATE OF } \\
\text { ISSUE }\end{array}$ & INFERENCE \\
\hline 1. & $\begin{array}{c}\text { Curvature-based } \\
\text { Selection of Feature by } \\
\text { applying in Classification } \\
\text { of Electronic Health Records }\end{array}$ & $\begin{array}{l}\text { Zheming, Jie Li, } \\
\text { NouraMoubayed. }\end{array}$ & 10 January 2021 & $\begin{array}{l}\text { Curvature-based Feature } \\
\text { Selection method - lack of } \\
\text { expert knowledge of clinical science, } \\
\text { visualisation the results of feature } \\
\text { ranking of the proposed CFS to } \\
\text { support better explainability. }\end{array}$ \\
\hline \multirow[t]{3}{*}{2.} & $\begin{array}{c}\text { Deep Neural Networks } \\
\text { Performance in Screening } \\
\text { of Breast Cancer. }\end{array}$ & $\begin{array}{l}\text { Nan Wub, Jason } \\
\text { Phangb, Jungkyu } \\
\text { Parkb }\end{array}$ & 20 March 2019 & $\begin{array}{l}\text { Deep convolutional neural } \\
\text { network; AUC of 0.895; high } \\
\text { accuracy-two-stage training } \\
\text { procedure, which allows them } \\
\text { to use a very high-capacity } \\
\text { patch-level network to learn } \\
\text { from pixel- level labels alongside } \\
\text { a network learning from } \\
\text { macroscopic breast-level labels. }\end{array}$ \\
\hline & & Habib Dhahri & 3November 2019 & $\begin{array}{l}\text { Geneticprogramming technique } \\
\text { for feature selection } \\
\text { and selection of parameter }\end{array}$ \\
\hline & $\begin{array}{l}\text { Machine Learning based } \\
\text { Automated Breast Cancer } \\
\text { Diagnosis algorithm }\end{array}$ & & & $\begin{array}{c}\text { values of classifiers of } \\
\text { machine learning. The Performance } \\
\text { was based on sensitivity, } \\
\text { specificity,precision, accuracy. }\end{array}$ \\
\hline 4. & $\begin{array}{c}\text { Prediction and detection } \\
\text { of breast cancer } \\
\text { using machine }\end{array}$ & Anoy Chowdhury & 19 June 2020 & $\begin{array}{l}\text { breast cancer tumour predictor } \\
\text { on the Wisconsin dataset } \\
\text { and created graphs and } \\
\text { results for the sam.e Selection } \\
\text { of appropriate algorithms with } \\
\text { good home dataset will lead } \\
\text { to the development of prediction } \\
\text { systems. Proper diagnosis of } \\
\text { patients suffering from breast } \\
\text { cancer can been sured } \\
\text { using the sesystems. }\end{array}$ \\
\hline 5. & $\begin{array}{l}\text { Applications of Machine } \\
\text { Learning in Cancer }\end{array}$ & $\begin{array}{l}\text { Joseph A, } \\
\text { David S. }\end{array}$ & 2006 & $\begin{array}{l}\text { From this paper it is concluded } \\
\text { that machine learning methods }\end{array}$ \\
\hline
\end{tabular}




\begin{tabular}{|c|c|c|c|c|}
\hline & $\begin{array}{l}\text { Prediction and } \\
\text { Prognosis }\end{array}$ & & & $\begin{array}{l}\text { can be used to substantially } \\
\text { (15-25\%) improvethe accuracy of } \\
\text { Predicting cancer } \\
\text { susceptibility, recurrence } \\
\text { and mortality. }\end{array}$ \\
\hline 6. & $\begin{array}{l}\text { Machine learning methods } \\
\text { for Breast cancer diagnostics }\end{array}$ & $\begin{array}{l}\text { Shahnorbanun, } \\
\text { Ashwaq Qasem. }\end{array}$ & 29January 2018 & $\begin{array}{l}\text { This paper deals with two most } \\
\text { popular techniques that use machine } \\
\text { earning (CADe \& CADx).False positive } \\
\text { rate is reduced significantly using } \\
\text { this method and rapidly improves } \\
\text { the prediction performance } \\
\text { of breast cancer. }\end{array}$ \\
\hline 7 & $\begin{array}{l}\text { Using Machine Learning } \\
\text { Algorithms for Breast Cancer } \\
\text { Risk Prediction and Diagnosis. }\end{array}$ & HibaAsria & 2016 & $\begin{array}{l}\text { In this paper, they have employed } \\
\text { four main algorithms: } \\
\text { SVM, NB, k-NN and C4.5 } \\
\text { on the Wiscons in Breast Cancer } \\
\text { (original) datasets; Compared } \\
\text { efficiency and effectiveness } \\
\text { of those algorithms in terms of } \\
\text { accuracy, precision, sensitivity and } \\
\text { specificity to find the best } \\
\text { classification accuracy; SVM reaches } \\
\text { accuracy of } 97.13 \% \text { and out } \\
\text { performother algorithms. }\end{array}$ \\
\hline 8. & $\begin{array}{l}\text { Early Detection of Breast } \\
\text { Cancer Using Machine } \\
\text { Learning Techniques. }\end{array}$ & Bashari Rad & September 2018 & $\begin{array}{c}\text { Breast cancer } \\
\text { AndML;introduced } \\
\text { in-depth literature } \\
\text { review on } \\
\text { ExistingML } \\
\text { methods;Suggest that } \\
\text { SVM- most popular } \\
\text { method used for cancer } \\
\text { detection applications. }\end{array}$ \\
\hline
\end{tabular}

\section{DISCUSSION AND CONCLUSION}

A large number of training sets based on breast level and pixel level labels are added to build a neural network for breast cancer classification on screening exams. The significant success is due to the amount of computation encapsulated in this model, that had been densely applied to the input images as an additional input channel to breast level model. The training to the model would be impossible with the current available hardware. Even Though our results are promising, we suggest he test set used are relatively small and are advised to require further clinical validations. We also acknowledge that our performance is much stronger than that of the specific task performed by radiologist.

Screening mammography is just the beginning step in the pipeline of diagnosis, further requiring the radiologist's final decision for another process and decision to biopsy after considering the mammography images and possible ultrasound. In our study, the hybrid model out performed both the neural network and expert radiologist suggestion, suggesting that the use of model like this could improve radiologist sensitivity for breast cancer classification and detection. Contrastingly, our model design is relatively simple. and with our model it is possible to develop more sophisticated and accurate models. Moreover, the prediction based on whether the patient had any visible cancer at mammography exam is the most simplest possible way of prediction among other. In addition to testing the model in real-time analyzing of screening mammograms, a transparent next step would be predicting the development of breast cancer in the future-at the earliest before it is even visible to a naked human eye and symptoms are well shown.

\section{REFERENCES}

Anuj Kumar Singh and Bhupendra Gupta "A novel approach for breast cancer detection and segmentation in mammography" Expert System With Applications 42(2015)990-1002.

Bellaachia Abdelghani and Erhan Guven, "Predicting carcinoma Survivability victimisation data processing Techniques”, Ninth Workshop on Mining Scientific and Engineering Datasets inconjunction with the Sixth Siam International Conference on data processing, 06.

Geras KJ, et al. (2017) High-resolution breast cancer 
screening of multi-view deep convolutional neural networks.

H. Blockeel and J. Struyf. economical algorithms for call tree cross-validation. Morgan Kaufmann,2001.

He K, Zhang X(2016) Deep residual learning for image recognition in CVPR.

He K, Zhang X (2016) Deep residual learning for image recognition.

Huang G, Liu Z, van der Maaten L, Weinberger KQ (2017) Densely connected convolutional networks in CVPR. J.Dheeba, N.Albert Singh "Computer-aided detection of breast cancer on mammograms: A swarm intelligence optimized wavelet neural network approach" Journal of Biomedical Informatics (2014).

J. Han and M. Kamber, "Data MiningTechniques", Morgan Kaufmann Publishers, 2000.

Krause J, et al. (2015) The unreasonable effectiveness of noisy data for fine-grained recognition.ar Xiv:1511.06789.
National Cancer Institute:http://www.cancer.gov/ cancertopics/types/breast.

National Cancer Institute Breast Cancer,http:// www.cancer.gov/cancertopics/types/breastcarcinoma Organization, http://www.breastcancer.org/symptoms/ types

Neeraj Bhargava, Girja Sharma, call Tree Analysis on J48 formula for knowledge Mining, June2013.

Oquab M, Bottou L, Laptev I, Sivic J (2014) Learning and transferring mid-level image representations using convolutional neural networks in CVPR.

William H Wolberg, OlviMangasarian, UCI Machine Learning Repository [http://archive.ics.uci.edu/ml]. $\mathrm{Wu}$ N, et al. (2019) The NYU breast cancer screening dataset Available at https://cs.nyu.edu/ kgeras/ reports/ datav1.0.pdf.

$\mathrm{Wu} \mathrm{N}$, et al. (2018) Breast density classification with deep convolutional neural networks in ICASSP. 